\title{
Strategic Alliance Typology and Survival Chances among Medium-Sized Manufacturing Firms in Tanzania
}

- Jeje Kafigi

\begin{abstract}
Firms strive to grow and survive. The survival chances can be fostered by a list of business strategies, such as strategic alliances. Different types of strategic alliances exist. This study groups the typology into manufacturing and non-manufacturing alliances. Little research has been done on Small and Medium Enterprises (SMEs), particularly Medium-Sized Enterprises (MEs), from the manufacturing industry in the least developed countries such as Tanzania in relation to the alliance typology and partnering firms' survival chances that can be explained by an ability to accumulate resources and reduce both costs and risks. A cross-sectional survey design and multistage probability sampling technique enabled the participation of 398 CEOs from three zones of Tanzania whose options were collected through questionnaires. In the context of this paper, both the Principal Component Analysis (PCA) and Multiple Regression Analysis (MRA) are applied to explain the two main alliance types and how they predict firms' survival chances respectively. To a great extent, we find that there exists a relationship between alliance typology and both the reduction of costs and risks, as well as resource accessibility. However, we argue that partners could adopt alliance types that concurrently reduce the costs and risks; but at the same time enabling them to acquire resources if they would carefully study and establish the link between costs, risks and resources.
\end{abstract}

Keywords: strategic alliances, alliance typology, survival chances, manufacturing medium-sized firms, principal component analysis, multiple regression analysis, Tanzania

JEL Classification: L14; L24; P13; P17

\section{INTRODUCTION}

Access to resources and ability to overcome both costs and risks are critical to any firm's success, growth and survival chances. However, these achievements are less likely to be met by individual firms, such as SMEs, that are generally constrained by a list of operational, financial and technological challenges. Most of these SMEs, including the medium-sized manufacturing firms in the least developed countries like Tanzania, cannot withstand the go-it-alone strategy. This is due to the fact that the strategy requires them to use their own resources, meet research and development costs, production, marketing, administrative and other operational costs. This cannot be met by a company whose capital ranges from say, TZS 200million to TZS 800 million coupled with limited access to finance, market, technology, information, and adequate working premises (The Government of the United Republic of Tanzania [URT], 2003). For these SMEs to address these 
operational hurdles, collaboration models seem to be the best alternative for them. These models include strategic alliances which are partnerships between autonomous firms that aim at gaining mutual benefits by jointly coordinating both tangible and intangible assets (Hagedoorn, Letterie, \& Palm, 2011; Todeva \& Knoke, 2005; Dussauge, Garrette, \& Mitchell, 2004; Das \& Teng, 2000; Elmuti \& Kathawala, 2001; Elmuti, Abebe, \& Nicolosi, 2005; Gulati, 1998; Knoke, 2009).

The strategic alliance typology is diverse. But the most common strategic alliance types can be in terms of buying and selling (Estélyiová, 2012), joint distribution (Coopers \& Lybrand, 1997; Išoraite, 2009; Mowla, 2012), and licensing agreements (Mowla, 2012; Zamir, Sahar, \& Zafar, 2014) such as product licensing (Išoraité, 2009) and technology licensing (Coopers \& Lybrand, 1997; Išoraite, 2009). Others include joint marketing agreements such as joint promotion (Coopers \& Lybrand, 1997; Mowla, 2012), research and development agreements (Coopers \& Lybrand, 1997; Išoraite, 2009; Zamir, Sahar, \& Zafar, 2014; Mowla, 2012), design collaboration and joint production (Coopers \& Lybrand, 1997). Others are such as franchising (Zamir, Sahar, \& Zafar, 2014; Išoraite, 2009), service agreements (Mowla, 2012), and outsourcing (Coopers and Lybrand, 1997; Išoraite, 2009; Zamir, Sahar, \& Zafar, 2014). Subcontracting, processing and assembling contracts are also included in the list (Estélyiová, 2012). Other types of strategic alliances include learning alliances whose objective is to reduce ignorance of partnering firms by providing information on markets, competencies, and new technologies. These alliances aim at capturing value from the joint learning activities and resources (Koza \& Lewin, 2000). Most of these alliances are in the form of ordinary contracts (Estélyiová, 2012). These contractual arrangements are always developing the simplest forms of strategic alliances (Mowla, 2012). They are created quickly to cater for the needs of a certain business opportunity (Estélyiová, 2012). Principally, they are short term agreements that are made when a formal structure of management is not needed (Zamir, Sahar, \& Zafar, 2014). They disband after the negotiated objective has been achieved (Estélyiová, 2012). That is why SMEs can opt for these contractual alliances due to affordable transaction costs of creating and dissolving (Estélyiová, 2012; Das \& Teng, 2000).

As we have already mentioned, firms' success heavily depend on resource accessibility, and both cost and risk reductions. Our assumption is that, the likelihood of accessing resources, and reducing both costs and risks depend on the type of strategic alliances formed by the partnering firms. We structure the alliance typology in two groups. There are those alliance types that are directly related to manufacturing and others which are indirectly related. Manufacturing alliances include joint production, design collaboration, technology licensing, collaboration in research and development, product licensing, assembling contracts and subcontracting. We define joint promotion, joint selling, joint distribution, learning alliances and service agreements as non-manufacturing alliances. The aim of this study is, therefore, to find whether the alliance typology fosters firms' survival chances. In this paper, we define survival chances as the likelihood of cost reduction, likelihood of risk reduction and likelihood of resource accessibility. Specifically, the study seeks to;

1. Find the influence of manufacturing alliances on the likelihood of cost reduction.

2. Find the influence of manufacturing alliances on the likelihood of resource accessibility.

3. Find the influence of non-manufacturing alliances on the likelihood of risk reduction. 


\section{THEORETICAL DEVELOPMENT}

\subsection{Manufacturing and Non-Manufacturing Activities}

The term manufacturing has been causing much confusion. In most cases, it has been used interchangeably with production. However, manufacturing and production are different. Basically, production is part of manufacturing (Livesey, 2006). The most used definition is the one that refers to manufacturing as processes that make parts and assemble them in machines. These processes include creating products from raw materials (Singh, 2006). On contrary, Livesey (2006) argues that the development, production and delivery of goods and services to customers are what constitute manufacturing. That is why Helper, Krueger, \& Wial (2012) refer to manufacturing as the major source of business innovation and that it plays a vital role in the development of the service sector. In this context, we find that development mentioned by Livesey (2006) includes several activities, such as research and designing. However, production remains to be a part of manufacturing whereby the conversion of raw material into finished goods using machine tools and manufacturing processes takes place (Singh, 2006). The inclusion of products delivery in the definition of Livesey (2006) above makes us argue that the re-packaging process, re-assembling, labelling and distribution are part of the manufacturing process. Livesey (2006) argues further that a firm is still a manufacturer even though it concentrates on marketing while its entire production plan is implemented by an outsourced firm. On the contrary, Singh (2006) points out that manufacturing cannot include distribution, neither handling nor storage of parts; since these activities are not directly related to transformation process of the particular parts. In this context, we can argue that other 'non-manufacturing activities', such as purchasing, distribution, logistics and inventory management constitute an operation process. IBM Corporation (2008) points out that a manufacturing firm depends greatly on operations to sell and deliver its products to the customers. In this paper, we relate manufacturing with production, designing, licensing, research and development, assembling contracts, and subcontracting (outsourcing). We also refer to activities, such as promotion, selling, distribution and other logistical arrangements, as non-manufacturing activities.

\subsection{Survival Chances and Alliance Typology}

The firm's survival depends greatly on the supply of resources (Sheppard, 1995). Generally, firms intend to gain access to the resources through strategic alliances (Al Khattab, 2012). For instance, they can use their contractual arrangements such as technology licensing to access new technology, significant technological information, and opportunities for technological transfer (Elmuti \& Kathawala, 2001; Hagedoorn \& Letterie \& Palm, 2011; Elmuti \& Abebe, \& Nicolosi, 2005); or adopt joint marketing agreements, such as joint promotion and joint distribution to access markets or future business (Elmuti \& Kathawala, 2001; Al Khattab, 2012; Chen $\&$ Tseng, 2005) as well as enhancing supply processes (Zineldin \& Dodourova, 2005). Firms' resources define the particular firms' strengths and weaknesses (Barney, 1991; Wernefelt, 1984). The firm's accumulated resources (Das \& Teng, 2000), such as skilled employees (Mills \& Platts, 2003; Wernefelt, 1984; Barney, 1991), factory building, machinery, efficient procedures and organizational processes, capital, business contacts, brand names, knowledge, firm attributes and information (Mills \& Platts, 2003; Barney, 1991; Wernefelt, 1984) build the firm's competitive strategy and eventually make it achieve competitive advantages (Das \& Teng, 2000). 
Sharing resources is one of the ways of accessing resources. Firms can share resources like capital, plants, distribution channels, patents and copyrights if they adopt alliance types, such as licensing, subcontracting, and distribution agreements. The sharing of other resources, like knowledge or opportunities for learning is possible if these firms adopt joint production, joint $R \& D$, and joint marketing and promotion (Das \& Teng, 2000). Again, these alliances help the partnering firms to share the costs of research and development (Elmuti \& Kathawala, 2001; Elmuti \& Abebe \& Nicolosi, 2005), reduce cost (Chen \& Tseng, 2005; Van Gils \& Zwart, 2009; Zineldin \& Dodourova, 2005) and minimize risks (Elmuti \& Kathawala, 2001). The firm's costs, regarded in this paper, are those related to the manufacturing process (including the actual production) and operation activities, such as storage, distribution, marketing, finance and other logistical costs. The assumption here is that, these costs are likely to be reduced when two or more partners collaborate. Also, in this paper, we regard risks as all kinds of risks whether systematic or non-systematic whose reduction can be fostered by the partnering firms' decision to form strategic alliances. Once these risks are clearly understood by the partners (Paik, 2005), they can be mitigated by various forms of strategic alliances, such as buying and selling, joint distribution, licensing agreements, joint marketing agreements, research and development agreements, design collaboration and production, franchising, service agreements, outsourcing, subcontracting, processing, and assembling. Apart from reducing risks, they can also reduce costs and uncertainties (Chen \& Tseng, 2005; Van Gils \& Zwart, 2009; Zineldin \& Dodourova, 2005; Elmuti \& Kathawala, 2001; Al Khattab, 2012; Hagedoorn, Letterie, \& Palm, 2011; Elmuti, Abebe, \& Nicolosi, 2005).

Firms expect their alliances to accelerate innovation in their products (Elmuti, Abebe, \& Nicolosi, 2005) and increase their productive capacities (Al Khattab, 2012); that is why partnering firms use different forms of strategic alliance to play down transaction and production costs (Martins, Serra, Leite, Ferreira, \& Li, 2010). These innovation and productive capacities can fuel competitive advantages, intensify competitive positioning and attain new efficiencies and competencies. However, these new efficiencies and competencies cannot be achieved unless the firms' resources are well managed (Ireland, Hoskisson, \& Hitt, 2009). These efficiencies and competencies can also be achieved should the costs and risks be managed. Since the intention of alliance partners is to reduce cost and risks (Chen \& Tseng, 2005; Van Gils \& Zwart, 2009; Zineldin \& Dodourova, 2005; Elmuti \& Kathawala, 2001), the attainment of their objectives depends greatly on the form of alliance entered. Resources, costs and risks relate. Alliance types that enable partners to acquire resources and minimize both costs and risks are those related to manufacturing and non-manufacturing as it has already been explained. We argue that these collaborations related to production, manufacturing design, technology, research and development, assembling, marketing, learning, subcontracting and all kinds of services, such as administration, communication, and other logistics, are likely to reduce costs and risks; and hence enable partners acquire resources. In this context, we analyse the influence of manufacturing alliances on the likelihood of cost reduction by proposing that;

- There is a relationship between cost reduction and joint production.

- There is a relationship between cost reduction and design collaboration.

- There is a relationship between cost reduction and technology licensing. 
- There is a relationship between cost reduction and collaboration in research and development.

- There is a relationship between cost reduction and product licensing.

- There is a relationship between cost reduction and assembling contracts.

- There is a relationship between cost reduction and subcontracting.

We also find the influence of manufacturing alliances on the likelihood of resource accessibility by proposing that;

- There is a relationship between resource accessibility and joint production.

- There is a relationship between resource accessibility and design collaboration.

- There is a relationship between resource accessibility and technology licensing.

- There is a relationship between resource accessibility and collaboration in research and development.

- There is a relationship between resource accessibility and product licensing.

- There is a relationship between resource accessibility and assembling contracts.

- There is a relationship between resource accessibility and subcontracting.

Finally, based on the influence of non-manufacturing alliances on the likelihood of risk reduction, we propose that;

- There is a relationship between risk reduction and joint promotion.

- There is a relationship between risk reduction and joint selling.

- There is a relationship between risk reduction and joint distribution.

- There is a relationship between risk reduction and learning alliances.

- There is a relationship between risk reduction and service agreements.

\section{METHODOLOGY}

\subsection{The Study Area and the Target Population}

All the regions of Tanzania Mainland which are classified into eight geographic zones were involved in this study. These eight zones are Western: (Tabora, Kigoma), Northern: (Kilimanjaro, Tanga, Arusha), Central: (Dodoma, Singida, Manyara), Southern Highlands: (Njombe, Iringa, Ruvuma), Lake: (Kagera, Mwanza, Mara, Shinyanga, Geita, Simiyu), Eastern: (Dar es Salaam, Pwani, Morogoro), Southern: (Lindi, Mtwara) and Southwest Highlands: (Rukwa, Katavi, Mbeya). We developed a list of manufacturing MEs from Small Industries Development Organization (SIDO), Tanzania Food and Drugs Authority (TFDA), and Business Registrations and Licensing Agency (BRELA) since the total number of manufacturing MEs in Tanzania was not in place by the time the research was conducted. These are those dealing with food and beverages; textiles and leather; wood and wood products; paper and paper products; chemical, petroleum and plastic products; pottery, glass and non-metallic products; basic metal industries; 
and fabricated metal products, machinery and equipment (National Bureau of Statistics [NBS], 2013). Although MEs as those employing between 50 and 99 people or use capital investment between Tshs 200 million and Tshs 800 million (URT, 2003), we define manufacturing MEs based on the number of employees.

\subsection{Sample Size and Sampling Procedure}

We assumed that about $50 \%$ of the manufacturing MEs operate aiming at obtaining the optimum number of manufacturing MEs (n) sampled (Cochran, 1977). We also set the committed error of $4.45 \%, \alpha$ of $5 \%$. Other values include $\mathrm{p}=0.5, \mathrm{q}=0.5$ and $\mathrm{Z}_{\alpha / 2}=1.96$. We computed $\mathrm{n}$ as;

$\mathrm{n}=\frac{Z^{2} \alpha / 2 \mathrm{pq}}{e^{2}}(1)$

The above formula gave us the optimum number of manufacturing MEs of 485 .

The study applied a cross-sectional survey design. We stratified all the regions of Tanzania into eight (8) zones as shown above. Using a multistage probability sampling technique, we purposively selected five zones and randomly chose three zones: Eastern, Northern and Lake. Thereafter, a systematic sampling was adopted to select the required number of manufacturing MEs from each zone through Probability Proportional to Size (PPS). This drew us 287, 103 and 95 MEs from Eastern, Northern and Lake Zones respectively.

\subsection{Data Collection and Analysis}

A total of 485 CEOs representing 485 manufacturing MEs were given 485 questionnaires. Each was required to fill in one questionnaire. The response rates from Eastern Zone, Northern Zone and Lake Zone were $85 \%, 84.5 \%$ and $70.5 \%$ respectively. This makes a total of 398 questionnaires that were both filled in and returned thus making a response rate of $82 \%$. The data were collected from November 2013 to May 2014. The types of strategic alliances explained both the non-manufacturing and manufacturing alliances through a Principal Component Analysis while the likelihood of resource accessibility and the reduction of both costs and risks were predicted by a Multiple Regression Analysis, aided by PASW 16 (SPSS 16).

\section{RESULTS}

Before predicting the influence of the types of strategic alliances on the likelihood of cost reduction, risk reduction and resource accessibility through MRA, we first employed the PCA to find whether there were relationships between the different types of strategic alliances and their corresponding two categories: the manufacturing and non-manufacturing alliances. We had a total of twelve independent variables: joint production $\left(\mathrm{X}_{\mathrm{ppr}}\right)$, design collaboration $\left(\mathrm{X}_{\mathrm{dcb}}\right)$, technology licensing $\left(\mathrm{X}_{\mathrm{tl}}\right)$, collaboration in research and development $\left(\mathrm{X}_{\mathrm{rdv}}\right)$, product licensing $\left(\mathrm{X}_{\mathrm{plc}}\right)$, assembling contracts $\left(\mathrm{X}_{\mathrm{ac}}\right)$ and subcontracting $\left(\mathrm{X}_{\mathrm{sb}}\right)$. Others included joint promotion $\left(\mathrm{X}_{\mathrm{pm}}\right)$, joint selling $\left(\mathrm{X}_{\mathrm{js}}\right)$ joint distribution $\left(\mathrm{X}_{\mathrm{ds}}\right)$, learning alliances $\left(\mathrm{X}_{\mathrm{lal}}\right)$ and service agreements $\left(\mathrm{X}_{\mathrm{sar}}\right)$. 
Tab. 1 - Correlation matrix with all variables. Source: Own

\begin{tabular}{|c|c|c|c|c|c|c|c|c|c|c|c|c|}
\hline Variable & $\mathrm{X}_{\mathrm{pm}}$ & $\mathrm{X}_{\mathrm{jsl}}$ & $\mathrm{X}_{\mathrm{jpr}}$ & $\mathrm{X}_{\mathrm{dst}}$ & $\mathrm{X}_{\mathrm{lal}}$ & $\mathrm{X}_{\mathrm{rdv}}$ & $\mathrm{X}_{\mathrm{dcb}}$ & $\mathrm{X}_{\mathrm{plc}}$ & $\mathrm{X}_{\mathrm{tlc}}$ & $\mathrm{X}_{\mathrm{sar}}$ & $\mathrm{X}_{\mathrm{sbc}}$ & $\mathrm{X}_{\text {act }}$ \\
\hline $\mathrm{X}_{\mathrm{ipm}}$ & 1.000 & & & & & & & & & & & \\
\hline $\mathrm{X}_{\mathrm{js}}$ & -.104 & 1.000 & & & & & & & & & & \\
\hline $\mathrm{X}_{\mathrm{ppr}}$ & .106 & -.272 & 1.000 & & & & & & & & & \\
\hline $\mathrm{X}_{\mathrm{dst}}$ & -.218 & .629 & -.149 & 1.000 & & & & & & & & \\
\hline $\mathrm{X}_{\mathrm{lal}}$ & .238 & .089 & .193 & .088 & 1.000 & & & & & & & \\
\hline $\mathrm{X}_{\mathrm{rdv}}$ & -.147 & .178 & .267 & .200 & .393 & 1.000 & & & & & & \\
\hline $\mathrm{X}_{\mathrm{dcb}}$ & -.033 & .877 & -.154 & .597 & .156 & .219 & 1.000 & & & & & \\
\hline $\mathrm{X}_{\mathrm{plc}}$ & -.027 & .223 & .162 & .219 & .276 & .383 & .243 & 1.000 & & & & \\
\hline $\mathrm{X}_{\text {tlc }}$ & -.011 & .257 & .177 & .245 & .161 & .309 & .229 & .612 & 1.000 & & & \\
\hline $\mathrm{X}_{\mathrm{sar}}$ & .251 & .163 & -.155 & .064 & .089 & .178 & .217 & .020 & .104 & 1.000 & & \\
\hline $\mathrm{X}_{\mathrm{sbc}}$ & -.079 & .319 & .135 & .220 & .202 & .286 & .280 & .389 & .452 & .070 & 1.000 & \\
\hline $\mathrm{X}_{\mathrm{act}}$ & .302 & -.234 & .052 & -.215 & .139 & .142 & -.224 & .005 & .107 & .530 & .055 & 1.000 \\
\hline
\end{tabular}

The PCA was run with all variables and it was found from the correlation matrix (Table 1) that "joint production" did not have at least one correlation with another variable; where $r \geq 0.3$. Therefore, we decided to remove the particular variable and re-run the analysis.

Tab. 2 - Correlation matrix $\left(\mathrm{X}_{\mathrm{ipr}}\right.$ removed). Source: Own

\begin{tabular}{|c|c|c|c|c|c|c|c|c|c|c|c|}
\hline Variable & $\mathrm{X}_{\mathrm{jpm}}$ & $\mathrm{X}_{\mathrm{js} \mathrm{l}}$ & $\mathrm{X}_{\mathrm{dst}}$ & $\mathrm{X}_{\mathrm{lal}}$ & $\mathrm{X}_{\mathrm{rdv}}$ & $\mathrm{X}_{\mathrm{dcb}}$ & $\mathrm{X}_{\mathrm{plc}}$ & $\mathrm{X}_{\mathrm{tlc}}$ & $\mathrm{X}_{\mathrm{sar}}$ & $\mathrm{X}_{\mathrm{sbc}}$ & $\mathrm{X}_{\text {act }}$ \\
\hline$X_{i p m}$ & 1.000 & & & & & & & & & & \\
\hline $\mathrm{X}_{\mathrm{js} 1}$ & -.104 & 1.000 & & & & & & & & & \\
\hline $\mathrm{X}_{\mathrm{dst}}$ & -.218 & .629 & 1.000 & & & & & & & & \\
\hline $\mathrm{X}_{\mathrm{lal}}$ & .238 & .089 & .088 & 1.000 & & & & & & & \\
\hline $\mathrm{X}_{\mathrm{rdv}}$ & -.147 & .178 & .200 & .393 & 1.000 & & & & & & \\
\hline $\mathrm{X}_{\mathrm{dcb}}$ & -.033 & .877 & .597 & .156 & .219 & 1.000 & & & & & \\
\hline $\mathrm{X}_{\mathrm{plc}}$ & -.027 & .223 & .219 & .276 & .383 & .243 & 1.000 & & & & \\
\hline $\mathrm{X}_{\text {tlc }}$ & -.011 & .257 & .245 & .161 & .309 & .229 & .612 & 1.000 & & & \\
\hline $\mathrm{X}_{\mathrm{sar}}$ & .251 & .163 & .064 & .089 & .178 & .217 & .020 & .104 & 1.000 & & \\
\hline $\mathrm{X}_{\mathrm{sbc}}$ & -.079 & .319 & .220 & .202 & .286 & .280 & .389 & .452 & .070 & 1.000 & \\
\hline $\mathrm{X}_{\mathrm{act}}$ & .302 & -.234 & -.215 & .139 & .142 & -.224 & .005 & .107 & .530 & .055 & 1.000 \\
\hline
\end{tabular}

The PCA was re-run. According to Table 2, all variables had at least one correlation with another variable; where $r \geq 0.3$. However, "joint promotion" was removed due to a KMO value of less than 0.5; as shown in Table 3 despite the fact that the overall KMO measure was 0.689, which is mediocre; on Kaiser's (1974) classification of measure values as shown in Table 4. 
Tab. 3 - Anti-image matrices ( $\mathrm{X}_{\mathrm{jpr}}$ removed). Source: Own

\begin{tabular}{|c|c|c|c|c|c|c|c|c|c|c|c|c|}
\hline \multirow{12}{*}{ 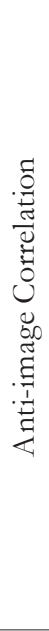 } & $\begin{array}{l}\text { Vari- } \\
\text { able }\end{array}$ & $\mathrm{X}_{\mathrm{jpm}}$ & $\mathrm{X}_{\mathrm{jsl}}$ & $\mathrm{X}_{\mathrm{dst}}$ & $\mathrm{X}_{\text {lal }}$ & $\mathrm{X}_{\mathrm{rdv}}$ & $\mathrm{X}_{\mathrm{dcb}}$ & $X_{\text {plc }}$ & $\mathrm{X}_{\mathrm{tlc}}$ & $\mathrm{X}_{\mathrm{sar}}$ & $\mathrm{X}_{\mathrm{sbc}}$ & $\mathrm{X}_{\text {act }}$ \\
\hline & $\mathrm{X}_{\mathrm{ipm}}$ & $.497 a$ & & & & & & & & & & \\
\hline & $\mathrm{X}_{\text {is } 1}$ & .060 & $.684^{a}$ & & & & & & & & & \\
\hline & $\mathrm{X}_{\mathrm{dst}}$ & .190 & -.238 & $.894^{a}$ & & & & & & & & \\
\hline & $\mathrm{X}_{\mathrm{lal}}$ & -.315 & .067 & -.052 & $.596^{a}$ & & & & & & & \\
\hline & $\mathrm{X}_{\mathrm{rdv}}$ & .299 & .057 & -.027 & -.356 & $.697^{a}$ & & & & & & \\
\hline & $\mathrm{X}_{\mathrm{dcb}}$ & -.123 & -.773 & -.126 & -.087 & -.084 & $.669^{a}$ & & & & & \\
\hline & $\mathrm{X}_{\mathrm{plc}}$ & -.016 & .058 & -.012 & -.123 & -.181 & -.077 & $.733^{a}$ & & & & \\
\hline & $\mathrm{X}_{\mathrm{tlc}}$ & -.048 & -.080 & -.092 & .083 & -.057 & .059 & -.505 & $.715^{\mathrm{a}}$ & & & \\
\hline & $\mathrm{X}_{\mathrm{sar}}$ & -.139 & -.010 & -.004 & .102 & -.115 & -.209 & .068 & .000 & $.543^{a}$ & & \\
\hline & $\mathrm{X}_{\mathrm{sbc}}$ & .088 & -.146 & .044 & -.094 & -.062 & .010 & -.103 & -.250 & .047 & $.850^{a}$ & \\
\hline & $\mathrm{X}_{\text {act }}$ & -.172 & .039 & .044 & -.065 & -.110 & .196 & .053 & -.110 & -.556 & -.091 & .579 a \\
\hline
\end{tabular}

a. Measures of Sampling Adequacy (MSA)

Tab. 4 - KMO and Bartlett's Test ( $\mathrm{X}_{\mathrm{jpr}}$ removed). Source: Own

\begin{tabular}{|c|c|c|}
\hline \multicolumn{2}{|c|}{ Kaiser-Meyer-Olkin Measure of Sampling Adequacy. } & .689 \\
\hline \multirow{3}{*}{ Bartlett's Test of Sphericity } & Approx. Chi-Square & 1685 \\
\cline { 2 - 3 } & $\mathrm{df}$ & 55 \\
\cline { 2 - 3 } & Sig. & .000 \\
\hline
\end{tabular}

Again, after removing “joint promotion”, the PCA was re-run and all variables had at least one correlation with another variable; where $r \geq 0.3$ as shown in Table 5 .

Tab. 5 - Correlation matrix $\left(\mathrm{X}_{\mathrm{jpr}}\right.$ and $\mathrm{X}_{\mathrm{jpm}}$ removed). Source: Own

\begin{tabular}{|c|c|c|c|c|c|c|c|c|c|c|}
\hline Variable & $\mathrm{X}_{\mathrm{jsl}}$ & $\mathrm{X}_{\mathrm{dst}}$ & $\mathrm{X}_{\mathrm{lal}}$ & $\mathrm{X}_{\mathrm{rdv}}$ & $\mathrm{X}_{\mathrm{dcb}}$ & $\mathrm{X}_{\mathrm{plc}}$ & $\mathrm{X}_{\mathrm{tcc}}$ & $\mathrm{X}_{\mathrm{sar}}$ & $\mathrm{X}_{\mathrm{sbc}}$ & $\mathrm{X}_{\mathrm{act}}$ \\
\hline $\mathrm{X}_{\mathrm{jsl}}$ & 1.000 & & & & & & & & & \\
\hline $\mathrm{X}_{\mathrm{dst}}$ & .629 & 1.000 & & & & & & & & \\
\hline $\mathrm{X}_{\mathrm{lal}}$ & .089 & .088 & 1.000 & & & & & & & \\
\hline $\mathrm{X}_{\mathrm{rdv}}$ & .178 & .200 & .393 & 1.000 & & & & & & \\
\hline $\mathrm{X}_{\mathrm{dcb}}$ & .877 & .597 & .156 & .219 & 1.000 & & & & & \\
\hline $\mathrm{X}_{\mathrm{plc}}$ & .223 & .219 & .276 & .383 & .243 & 1.000 & & & & \\
\hline $\mathrm{X}_{\mathrm{tlc}}$ & .257 & .245 & .161 & .309 & .229 & .612 & 1.000 & & & \\
\hline $\mathrm{X}_{\mathrm{sar}}$ & .163 & .064 & .089 & .178 & .217 & .020 & .104 & 1.000 & & \\
\hline $\mathrm{X}_{\mathrm{sbc}}$ & .319 & .220 & .202 & .286 & .280 & .389 & .452 & .070 & 1.000 & \\
\hline $\mathrm{X}_{\mathrm{act}}$ & -.234 & -.215 & .139 & .142 & -.224 & .005 & .107 & .530 & .055 & 1.000 \\
\hline
\end{tabular}


However, after examining the anti-image matrices (Table 6), we realized that "service agreements" had to be removed; since its KMO was less than 0.5. Again, the overall KMO measure was 0.706, which is middling; on Kaiser's (1974) classification of measure values as shown in Table 7.

Tab. 6 - Anti-image matrices $\left(\mathrm{X}_{\mathrm{jpr}}\right.$ and $\mathrm{X}_{\mathrm{jpm}}$ removed). Source: Own

\begin{tabular}{|c|c|c|c|c|c|c|c|c|c|c|c|}
\hline \multirow{11}{*}{ 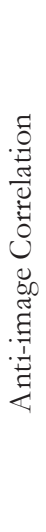 } & Variable & $\mathrm{X}_{\mathrm{js}}$ & $\mathrm{X}_{\mathrm{dst}}$ & $\mathrm{X}_{\mathrm{lal}}$ & $\mathrm{X}_{\mathrm{rdv}}$ & $\mathrm{X}_{\mathrm{dcb}}$ & $\mathrm{X}_{\mathrm{plc}}$ & $\mathrm{X}_{\mathrm{tlc}}$ & $\mathrm{X}_{\mathrm{sar}}$ & $\mathrm{X}_{\mathrm{sbc}}$ & $\mathrm{X}_{\text {act }}$ \\
\hline & $\mathrm{X}_{\mathrm{jsl}}$ & $.680^{\mathrm{a}}$ & & & & & & & & & \\
\hline & $\mathrm{X}_{\mathrm{dst}}$ & -.255 & $.910^{a}$ & & & & & & & & \\
\hline & $\mathrm{X}_{\mathrm{lal}}$ & .091 & .008 & $.698^{a}$ & & & & & & & \\
\hline & $\mathrm{X}_{\mathrm{rdv}}$ & .041 & -.089 & -.289 & $.812^{a}$ & & & & & & \\
\hline & $\mathrm{X}_{\mathrm{dcb}}$ & -.773 & -.106 & -.134 & -.050 & $.672^{\mathrm{a}}$ & & & & & \\
\hline & $\mathrm{X}_{\mathrm{plc}}$ & .059 & -.009 & -.135 & -.185 & -.080 & $.730^{a}$ & & & & \\
\hline & $\mathrm{X}_{\mathrm{tlc}}$ & -.077 & -.084 & .071 & -.045 & .054 & -.506 & $.717^{a}$ & & & \\
\hline & $\mathrm{X}_{\mathrm{sar}}$ & -.002 & .023 & .062 & -.078 & -.231 & .067 & -.007 & $.494^{a}$ & & \\
\hline & $\mathrm{X}_{\mathrm{sbc}}$ & -.152 & .027 & -.070 & -.093 & .021 & -.102 & -.247 & .060 & $.858^{a}$ & \\
\hline & $\mathrm{X}_{\mathrm{act}}$ & .050 & .080 & -.127 & -.062 & .179 & .051 & -.121 & -.594 & -.077 & $.526^{\mathrm{a}}$ \\
\hline
\end{tabular}

a. Measures of Sampling Adequacy (MSA)

Tab. 7 - KMO and Bartlett's Test ( $\mathrm{X}_{\mathrm{jpr}}$ and $\mathrm{X}_{\mathrm{jpm}}$ removed). Source: Own

\begin{tabular}{|c|c|c|}
\hline \multicolumn{2}{|c|}{ Kaiser-Meyer-Olkin Measure of Sampling Adequacy. } & .706 \\
\hline \multirow{3}{*}{ Bartlett's Test of Sphericity } & Approx. Chi-Square & 1559 \\
\cline { 2 - 3 } & $\mathrm{df}$ & 45 \\
\cline { 2 - 3 } & Sig. & .000 \\
\hline
\end{tabular}

Again, after removing "service agreements", the PCA was re-run and "assembling contracts" did not have at least one correlation with another variable; where $r \geq 0.3$ as shown in Table 8 and therefore was removed.

Tab. 8 - Correlation matrix $\left(\mathrm{X}_{\mathrm{ppr}}, \mathrm{X}_{\mathrm{ppm}}\right.$ and $\mathrm{X}_{\mathrm{sar}}$ removed). Source: Own

\begin{tabular}{|c|c|c|c|c|c|c|c|c|c|}
\hline Variable & $\mathrm{X}_{\mathrm{jsl}}$ & $\mathrm{X}_{\mathrm{dst}}$ & $\mathrm{X}_{\mathrm{lal}}$ & $\mathrm{X}_{\mathrm{rdv}}$ & $\mathrm{X}_{\mathrm{dcb}}$ & $\mathrm{X}_{\mathrm{plc}}$ & $\mathrm{X}_{\mathrm{tlc}}$ & $\mathrm{X}_{\mathrm{sbc}}$ & $\mathrm{X}_{\mathrm{act}}$ \\
\hline $\mathrm{X}_{\mathrm{jsl}}$ & 1.000 & & & & & & & & \\
\hline $\mathrm{X}_{\mathrm{dst}}$ & .629 & 1.000 & & & & & & & \\
\hline $\mathrm{X}_{\mathrm{lal}}$ & .089 & .088 & 1.000 & & & & & & \\
\hline $\mathrm{X}_{\mathrm{rdv}}$ & .178 & .200 & .393 & 1.000 & & & & & \\
\hline $\mathrm{X}_{\mathrm{dcb}}$ & .877 & .597 & .156 & .219 & 1.000 & & & & \\
\hline $\mathrm{X}_{\mathrm{plc}}$ & .223 & .219 & .276 & .383 & .243 & 1.000 & & & \\
\hline $\mathrm{X}_{\mathrm{tlc}}$ & .257 & .245 & .161 & .309 & .229 & .612 & 1.000 & & \\
\hline $\mathrm{X}_{\mathrm{sbc}}$ & .319 & .220 & .202 & .286 & .280 & .389 & .452 & 1.000 & \\
\hline $\mathrm{X}_{\mathrm{act}}$ & -.234 & -.215 & .139 & .142 & -.224 & .005 & .107 & .055 & 1.000 \\
\hline
\end{tabular}


The PCA was re-run and all variables had at least one correlation with another variable where $r$ $\geq 0.3$ as shown in Table 9 .

Tab. 9 - Correlation matrix $\left(\mathrm{X}_{\mathrm{jpr}}, \mathrm{X}_{\mathrm{ppm}}, \mathrm{X}_{\mathrm{sar}}\right.$ and $\mathrm{X}_{\mathrm{act}}$ removed). Source: Own

\begin{tabular}{|c|c|c|c|c|c|c|c|c|}
\hline Variable & $\mathrm{X}_{\mathrm{jsl}}$ & $\mathrm{X}_{\mathrm{dst}}$ & $\mathrm{X}_{\mathrm{lal}}$ & $\mathrm{X}_{\mathrm{rdv}}$ & $\mathrm{X}_{\mathrm{dcb}}$ & $\mathrm{X}_{\mathrm{plc}}$ & $\mathrm{X}_{\mathrm{tcc}}$ & $\mathrm{X}_{\mathrm{sbc}}$ \\
\hline $\mathrm{X}_{\mathrm{jsl}}$ & 1.000 & & & & & & & \\
\hline $\mathrm{X}_{\mathrm{dst}}$ & .629 & 1.000 & & & & & & \\
\hline $\mathrm{X}_{\mathrm{al}}$ & .089 & .088 & 1.000 & & & & & \\
\hline $\mathrm{X}_{\mathrm{rdv}}$ & .178 & .200 & .393 & 1.000 & & & & \\
\hline $\mathrm{X}_{\mathrm{dcb}}$ & .877 & .597 & .156 & .219 & 1.000 & & & \\
\hline $\mathrm{X}_{\mathrm{plc}}$ & .223 & .219 & .276 & .383 & .243 & 1.000 & & \\
\hline $\mathrm{X}_{\mathrm{tlc}}$ & .257 & .245 & .161 & .309 & .229 & .612 & 1.000 & \\
\hline $\mathrm{X}_{\mathrm{sbc}}$ & .319 & .220 & .202 & .286 & .280 & .389 & .452 & 1.000 \\
\hline
\end{tabular}

We also examined the anti-image matrices (Table 10); and realized that all variables had KMO values greater than 0.650 , which showed adequacy of sampling. Also, the overall KMO measure was 0.736, which is middling; on Kaiser's (1974) classification of measure values as shown in Table 11. We also found that Bartlett's Test of Sphericity was statistically significant $(\mathrm{p}<.05)$ as shown in Table 11.

Tab. 10 - Anti-image matrices $\left(\mathrm{X}_{\mathrm{ppr}}, \mathrm{X}_{\mathrm{jpm}}, \mathrm{X}_{\mathrm{sar}}\right.$ and $\mathrm{X}_{\mathrm{act}}$ removed). Source: Own

\begin{tabular}{|c|c|c|c|c|c|c|c|c|c|}
\hline \multirow{9}{*}{ 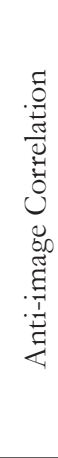 } & Variable & $\mathrm{X}_{\mathrm{js} \mathrm{l}}$ & $\mathrm{X}_{\mathrm{dst}}$ & $\mathrm{X}_{\text {lal }}$ & $\mathrm{X}_{\mathrm{rdv}}$ & $\mathrm{X}_{\mathrm{dcb}}$ & $\mathrm{X}_{\mathrm{plc}}$ & $\mathrm{X}_{\mathrm{tlc}}$ & $\mathrm{X}_{\mathrm{sbc}}$ \\
\hline & $\mathrm{X}_{\mathrm{js} \mathrm{I}}$ & $.654^{a}$ & & & & & & & \\
\hline & $\mathrm{X}_{\mathrm{dst}}$ & -.264 & $.910^{a}$ & & & & & & \\
\hline & $\mathrm{X}_{\text {lal }}$ & .099 & .020 & $.702^{a}$ & & & & & \\
\hline & $\mathrm{X}_{\mathrm{rdv}}$ & .050 & -.073 & -.305 & $.803^{a}$ & & & & \\
\hline & $\mathrm{X}_{\mathrm{dcb}}$ & -.801 & -.110 & -.118 & -.063 & $.670^{a}$ & & & \\
\hline & $\mathrm{X}_{\mathrm{plc}}$ & .053 & -.024 & -.129 & -.168 & -.073 & $.744^{a}$ & & \\
\hline & $X_{\text {tlc }}$ & -.069 & -.067 & .055 & -.068 & .063 & -.498 & $.723^{a}$ & \\
\hline & $\mathrm{X}_{\mathrm{sbc}}$ & -.149 & .032 & -.080 & -.096 & .039 & -.101 & -.259 & $.857^{\mathrm{a}}$ \\
\hline
\end{tabular}

a. Measures of Sampling Adequacy (MSA)

Tab. 11 - KMO and Bartlett's Test $\left(\mathrm{X}_{\mathrm{ppr}}, \mathrm{X}_{\mathrm{ppm}}, \mathrm{X}_{\mathrm{sar}}\right.$ and $\mathrm{X}_{\mathrm{act}}$ removed). Source: Own

\begin{tabular}{|c|c|c|}
\hline \multicolumn{2}{|c|}{ Kaiser-Meyer-Olkin Measure of Sampling Adequacy. } & .736 \\
\hline \multirow{3}{*}{ Bartlett's Test of Sphericity } & Approx. Chi-Square & 1292 \\
\cline { 2 - 3 } & df & 28 \\
\cline { 2 - 3 } & Sig. & .000 \\
\hline
\end{tabular}




\subsection{The Number of Components Retained}

We decided to retain two components since only two components had Eigenvalues greater than 1 as shown in Table 12. We also decided to retain components that could explain at least $60 \%$ or $70 \%$ of the total variance. Using the lower criterion of $60 \%$, we decided to retain the first two components as shown in Table 12.

Tab. 12 - Total variance explained. Source: Own

\begin{tabular}{|c|c|c|c|c|c|c|c|c|c|}
\hline \multirow{2}{*}{ 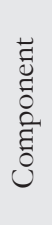 } & \multicolumn{3}{|c|}{ Initial Eigenvalues } & \multicolumn{3}{|c|}{$\begin{array}{l}\text { Extraction Sums of } \\
\text { Squared Loadings }\end{array}$} & \multicolumn{3}{|c|}{$\begin{array}{c}\text { Rotation Sums of Squared } \\
\text { Loadings }\end{array}$} \\
\hline & Total & $\begin{array}{c}\% \text { of } \\
\text { Variance }\end{array}$ & $\begin{array}{c}\text { Cumu- } \\
\text { lative } \\
\%\end{array}$ & Total & $\begin{array}{c}\% \text { of } \\
\text { Variance }\end{array}$ & $\begin{array}{l}\text { Cumu- } \\
\text { lative } \\
\%\end{array}$ & Total & $\begin{array}{c}\% \text { of } \\
\text { Variance }\end{array}$ & $\begin{array}{c}\text { Cumu- } \\
\text { lative } \\
\%\end{array}$ \\
\hline 1 & 3.250 & 40.628 & 40.628 & 3.250 & 40.628 & 40.628 & 2.462 & 30.774 & 30.774 \\
\hline 2 & 1.594 & 19.924 & 60.552 & 1.594 & 19.924 & 60.552 & 2.382 & 29.778 & 60.552 \\
\hline 3 & .986 & 12.319 & 72.871 & & & & & & \\
\hline 4 & .653 & 8.160 & 81.031 & & & & & & \\
\hline 5 & .579 & 7.243 & 88.274 & & & & & & \\
\hline 6 & .457 & 5.709 & 93.983 & & & & & & \\
\hline 7 & .365 & 4.561 & 98.544 & & & & & & \\
\hline 8 & .116 & 1.456 & 100.000 & & & & & & \\
\hline
\end{tabular}

Extraction Method: Principal Component Analysis.

Also, the visual inspection of the scree plot (see Figure 1) led to the retention of two components as well.

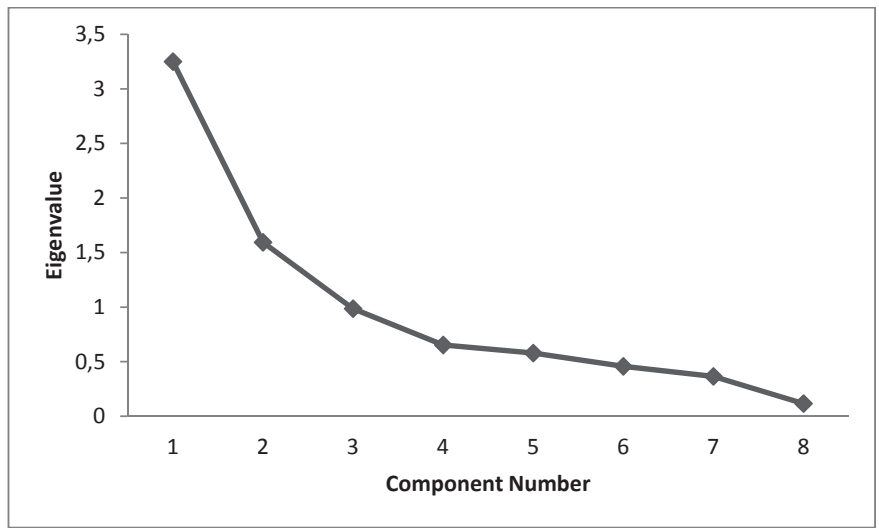

Fig. 1 - Screen plot. Source: Own

We also examined the Rotated Component Matrix (Table 13); and found that it appeared to be a simple structure. This is shown in Table 13 where each variable has only one component that loads strongly on it, and each component loads strongly on at least three variables. 
Tab. 13 - Rotated component matrixa. Source: Own

\begin{tabular}{|c|c|c|}
\hline \multirow{2}{*}{ Variable } & \multicolumn{2}{|c|}{ Component } \\
\cline { 2 - 3 } & 1 & 2 \\
\hline $\mathrm{X}_{\mathrm{jsl}}$ & .934 & \\
\hline $\mathrm{X}_{\mathrm{dst}}$ & .911 & \\
\hline $\mathrm{X}_{\mathrm{lal}}$ & .797 & \\
\hline $\mathrm{X}_{\mathrm{rdv}}$ & & .790 \\
\hline $\mathrm{X}_{\mathrm{dcb}}$ & & .735 \\
\hline $\mathrm{X}_{\mathrm{plc}}$ & & .676 \\
\hline $\mathrm{X}_{\mathrm{tlc}}$ & & .615 \\
\hline $\mathrm{X}_{\mathrm{sbc}}$ & & .568 \\
\hline
\end{tabular}

Extraction Method: Principal Component Analysis.

Rotation Method: Varimax with Kaiser Normalization.

a. Rotation converged in 3 iterations.

Since we had variables $\mathrm{X}_{\mathrm{ppr}}, \mathrm{X}_{\mathrm{dcb}}, \mathrm{X}_{\mathrm{tlc}}, \mathrm{X}_{\mathrm{rdv}}, \mathrm{X}_{\mathrm{plc}}, \mathrm{X}_{\mathrm{act}}$ and $\mathrm{X}_{\mathrm{sbc}}$ associated with manufacturing alliances and variables $\mathrm{X}_{\mathrm{jpm}}, \mathrm{X}_{\mathrm{js}}, \mathrm{X}_{\mathrm{dst}}, \mathrm{X}_{\mathrm{lal}}$ and $\mathrm{X}_{\mathrm{sar}}$ associated with non-manufacturing alliances, and we have components loading on some of these variables, it is, therefore, likely that Component 1 represents non-manufacturing alliances and Component 2 represents manufacturing alliances.

\subsection{Hypothesis Testing}

\section{Cost Reduction and Manufacturing Alliances}

In finding the relationship between manufacturing alliances and cost reduction, we tested the following hypotheses;

$\mathrm{H} 0: \omega_{\mathrm{dcb}}=0$

$\mathrm{H} 1: \omega_{\mathrm{dcb}} \neq 0$

$\mathrm{H} 0: \omega_{\mathrm{tlc}}=0$

$\mathrm{H} 1: \omega_{\mathrm{tlc}} \neq 0$

$\mathrm{H} 0: \omega_{\mathrm{rdv}}=0$

$\mathrm{H} 1: \omega_{\text {rdv }} \neq 0$

$\mathrm{H} 0: \omega_{\mathrm{plc}}=0$

$\mathrm{H} 1: \omega_{\mathrm{plc}} \neq 0$

$\mathrm{H} 0: \omega_{\mathrm{sbc}}=0$

$\mathrm{H} 1: \omega_{\mathrm{sbc}} \neq 0$

Where $\omega_{0}, \omega_{\mathrm{dcb}}, \omega_{\mathrm{tlc}}, \omega_{\mathrm{rdv}}, \omega_{\mathrm{plc}}$ and $\omega_{\mathrm{sbc}}$ are coefficients for Constant, $\mathrm{X}_{\mathrm{dcb}}, \mathrm{X}_{\mathrm{tlc}}, \mathrm{X}_{\mathrm{rdv}}, \mathrm{X}_{\mathrm{plc}}$ and $\mathrm{X}_{\mathrm{sbc}}$ respectively. 
$\mathrm{X}_{\mathrm{dcb}}$ denotes "design collaboration"

$\mathrm{X}_{\text {tlc }}$ denotes "technology licensing"

$\mathrm{X}_{\mathrm{rdv}}$ denotes "collaboration in research and development"

$\mathrm{X}_{\mathrm{plc}}$ denotes "product licensing"

$\mathrm{X}_{\mathrm{sbc}}$ denotes "subcontracting"

$\mathrm{Y}_{\text {lcr }}$ denotes "likelihood of cost reduction"

CEOs were asked to rate their level of agreement with $\mathrm{X}_{\mathrm{dcb}}, \mathrm{X}_{\mathrm{tc}}, \mathrm{X}_{\mathrm{rdv}}, \mathrm{X}_{\mathrm{plc}}$ and $\mathrm{X}_{\mathrm{sbc}}$ using a fivepoint scale (1. Strongly disagree, 2. Disagree, 3. Neither agree nor disagree, 4. Agree, 5. Strongly agree). They were also asked to rate $\mathrm{Y}_{\mathrm{lcr}}$ in percentage (0 to 100). From these variables, a multiple regression equation is formulated as

$\mathrm{Y}_{\mathrm{lcr}}=\omega_{0}+\omega_{\mathrm{dcb}} \mathrm{X}_{\mathrm{dcb}}+\omega_{\text {(tlc })} \mathrm{X}_{\mathrm{tc}}+\omega_{\mathrm{rdv}} \mathrm{X}_{\mathrm{rdv}}+\omega_{\mathrm{plc}} \mathrm{X}_{\mathrm{plc}}+\omega_{\mathrm{sbc}} \mathrm{X}_{\mathrm{sbc}}$

Tab. 14 - Multiple regression output for a linear relationship between Ylcr and Xdcb, Xtlc, Xrdv, Xplc, Xsbc. Source: Own

\begin{tabular}{|c|c|c|c|c|}
\hline \multicolumn{5}{|c|}{ Model Summary } \\
\hline Model & $\mathrm{R}$ & R Square & $\begin{array}{c}\text { Adjusted R } \\
\text { Square }\end{array}$ & $\begin{array}{c}\text { Std. Error of the } \\
\text { Estimate }\end{array}$ \\
\hline 1 & $.438^{\mathrm{a}}$ & .192 & .182 & 15.39063 \\
\hline
\end{tabular}

a. Predictors: (Constant), $\mathrm{X}_{\mathrm{dcb}}, \mathrm{X}_{\mathrm{tlc}}, \mathrm{X}_{\mathrm{rdv}}, \mathrm{X}_{\mathrm{plc}}, \mathrm{X}_{\mathrm{sbc}}$

\begin{tabular}{|c|c|c|c|c|c|c|}
\hline \multicolumn{9}{|c|}{ ANOVA $^{|c|}$} \\
\hline \multicolumn{2}{|c|}{ Model } & $\begin{array}{c}\text { Sum of } \\
\text { Squares }\end{array}$ & df & $\begin{array}{c}\text { Mean } \\
\text { Square }\end{array}$ & F & Sig. \\
\hline \multirow{3}{*}{1} & Regression & 22039.839 & 5 & 4407.968 & 18.609 & $.000^{\mathrm{a}}$ \\
\cline { 2 - 7 } & Residual & 92853.620 & 392 & 236.871 & & \\
\cline { 2 - 8 } & Total & 114893.460 & 397 & & & \\
\hline
\end{tabular}

a. Predictors: (Constant), $\mathrm{X}_{\mathrm{dcb}}, \mathrm{X}_{\mathrm{tlc}}, \mathrm{X}_{\mathrm{rdv}}, \mathrm{X}_{\mathrm{plc}}, \mathrm{X}_{\mathrm{sbc}}$

b. Dependent Variable: $Y_{\text {lcr }}$ 


\begin{tabular}{|c|c|c|c|c|c|c|c|c|}
\hline \multicolumn{9}{|c|}{ Coefficients ${ }^{a}$} \\
\hline & \multirow{2}{*}{ Model } & \multicolumn{2}{|c|}{$\begin{array}{c}\text { Unstandardized } \\
\text { Coefficients }\end{array}$} & \multirow{2}{*}{$\begin{array}{c}\begin{array}{r}\text { Standardized } \\
\text { Coefficients }\end{array} \\
\text { Beta }\end{array}$} & \multirow{2}{*}{$\mathrm{t}$} & \multirow{2}{*}{ Sig. } & \multicolumn{2}{|c|}{$\begin{array}{c}95 \% \text { Confidence } \\
\text { Interval for B }\end{array}$} \\
\hline & & B & $\begin{array}{l}\text { Std. } \\
\text { Error }\end{array}$ & & & & $\begin{array}{l}\text { Lower } \\
\text { Bound }\end{array}$ & $\begin{array}{l}\text { Upper } \\
\text { Bound }\end{array}$ \\
\hline \multirow{6}{*}{1} & (Constant) & 48.792 & 3.549 & & 13.747 & .000 & 41.814 & 55.770 \\
\hline & $\mathrm{X}_{\mathrm{dcb}}$ & 2.707 & .729 & .262 & 3.714 & .000 & 1.274 & 4.140 \\
\hline & $\mathrm{X}_{\mathrm{tlc}}$ & 2.227 & .755 & .202 & 2.948 & .003 & .742 & 3.712 \\
\hline & $\mathrm{X}_{\mathrm{rdv}}$ & 1.972 & .789 & .117 & 2.498 & .013 & .420 & 3.524 \\
\hline & $\mathrm{X}_{\mathrm{plc}}$ & 1.603 & .702 & .111 & 2.285 & .023 & .224 & 2.983 \\
\hline & $\mathrm{X}_{\mathrm{sbc}}$ & -4.142 & .581 & -.441 & -7.129 & .000 & -5.285 & -3.000 \\
\hline
\end{tabular}

a. Dependent Variable: $\mathrm{Y}_{\text {lcr }}$

From Table 14 we see that $\mathrm{X}_{-\mathrm{dcb}}, \mathrm{X}_{-\mathrm{tlc}}, \mathrm{X}_{-\mathrm{rdv}}, \mathrm{X}_{-\mathrm{plc}}$ and $\mathrm{X}_{-\mathrm{sbc}}$ statistically significantly predict $\mathrm{Y}_{\mathrm{lcr}}, \mathrm{F}(5,392)=18.609, \mathrm{p}<0.05$. We can also see that $\mathrm{X}_{-\mathrm{dcb}}, \mathrm{X}_{-\mathrm{tc}}, \mathrm{X}_{-\mathrm{rdv}}, \mathrm{X}_{-\mathrm{plc}}$ and $\mathrm{X}_{-\mathrm{sbc}}$ explain $19.2 \%$ of the variability of $\mathrm{Y}_{\mathrm{lcr}}$. We can therefore set a new regression equation as

$\mathrm{Y}_{\mathrm{lcr}}=48.792+2.707 \mathrm{X}_{\mathrm{dcb}}+2.227 \mathrm{X}_{\mathrm{tlc}}+1.972 \mathrm{X}_{\mathrm{rdv}}+1.603 \mathrm{X}_{\mathrm{plc}}-4.142 \mathrm{X}_{\mathrm{sbc}}$

From the first hypothesis, (H0: $\left.\omega_{\mathrm{dcb}}=0, \mathrm{H} 1: \omega_{\mathrm{dcb}} \neq 0\right)$, we reject $\mathrm{H} 0$; since $\omega_{\mathrm{dcb}}(2.707)$ is statistically significantly different from $0(\mathrm{p}<0.05)$. We find a positive relationship between $\mathrm{X}_{\mathrm{dcb}}$ and $\mathrm{Y}_{\mathrm{lcr}}$. Regarding the second hypothesis, $\left(\mathrm{H} 0: \omega_{\text {tlc }}=0, \mathrm{H} 1: \omega_{\mathrm{tlc}} \neq 0\right)$, we reject H0; since $\omega_{\text {tlc }}(2.227)$ is statistically significantly different from $0(\mathrm{p}<0.05)$. A positive relationship between $\mathrm{X}_{\mathrm{tlc}}$ and $\mathrm{Y}_{\mathrm{Icr}}$ is revealed. Again, from the third hypothesis, $\left(\mathrm{H} 0: \omega_{\mathrm{rdv}}=0, \mathrm{H} 1: \omega_{\mathrm{rdv}} \neq 0\right)$, we reject $\mathrm{H} 0$; since $\omega_{\text {rdv }}(1.972)$ is statistically significantly different from $0(\mathrm{p}<0.05)$. We also find a positive relationship between $X_{\text {rdv }}$ and $Y_{\text {lcr }}$. Regarding the fourth hypothesis, $\left(H 0: \omega_{\text {plc }}=0, H 1: \omega_{\text {plc }} \neq 0\right)$, we reject $\mathrm{H} 0$; since $\omega_{\text {plc }}(1.603)$ is statistically significantly different from $0(\mathrm{p}<0.05)$. Again, a positive relationship between $X_{\mathrm{plc}}$ and $Y_{\mathrm{lcr}}$ is found. From the fifth hypothesis, $\left(\mathrm{H} 0: \omega_{\mathrm{sbc}}=0, \mathrm{H} 1: \omega_{\mathrm{sbc}} \neq 0\right)$, we reject $\mathrm{H} 0$; since $\omega_{\mathrm{sbc}}(-4.142)$ is statistically significantly different from $0(\mathrm{p}<0.05)$ but a negative relationship between $\mathrm{X}_{\mathrm{sbc}}$ and $\mathrm{Y}_{\mathrm{lcr}}$ is found.

\section{Resource Accessibility and Manufacturing Alliances}

In finding the relationship between manufacturing alliances and resource accessibility, we tested the following hypotheses;

$$
\begin{aligned}
& \mathrm{H} 0: \omega_{\mathrm{dcb} 1}=0 \\
& \mathrm{H} 1: \omega_{\mathrm{dcb} 1} \neq 0 \\
& \mathrm{H} 0: \omega_{\mathrm{tcc} 1}=0 \\
& \mathrm{H} 1: \omega_{\mathrm{tlc1}} \neq 0 \\
& \mathrm{H} 0: \omega_{\mathrm{rdv} 1}=0 \\
& \mathrm{H} 1: \omega_{\mathrm{rdv} 1} \neq 0
\end{aligned}
$$


$\mathrm{H} 0: \omega_{\mathrm{plc} 1}=0$

$\mathrm{H} 1: \omega_{\text {plc1 }} \neq 0$

$\mathrm{H} 0: \omega_{\mathrm{sbc1}}=0$

$\mathrm{H} 1: \omega_{\mathrm{sbc} 1} \neq 0$

Where $\omega_{01}, \omega_{\mathrm{dcb} 1}, \omega_{\mathrm{tlc} 1}, \omega_{\mathrm{rdv} 1}, \omega_{\mathrm{plc} 1}$ and $\omega_{\mathrm{sbc1}}$ are coefficients for Constant, $\mathrm{X}_{\mathrm{dcb}}, \mathrm{X}_{\mathrm{tlc}}, \mathrm{X}_{\mathrm{rdv}}, \mathrm{X}_{\mathrm{plc}}$ and $\mathrm{X}_{\mathrm{sbc}}$ respectively (in relationship with $\mathrm{Y}_{\mathrm{lra}}$ )

$\mathrm{X}_{\mathrm{dcb}}$ denotes "design collaboration"

$\mathrm{X}_{\text {tlc }}$ denotes "technology licensing”

$\mathrm{X}_{\mathrm{rdv}}$ denotes "collaboration in research and development"

$\mathrm{X}_{\mathrm{plc}}$ denotes "product licensing"

$\mathrm{X}_{\mathrm{sbc}}$ denotes "subcontracting"

$\mathrm{Y}_{\text {lra }}$ denotes "likelihood of resource accessibility"

CEOs were asked to rate their level of agreement with $\mathrm{X}_{\mathrm{dcb}}, \mathrm{X}_{\mathrm{tc}}, \mathrm{X}_{\mathrm{rdv}}, \mathrm{X}_{\mathrm{plc}}$ and $\mathrm{X}_{\mathrm{sbc}}$ using a fivepoint scale (1. Strongly disagree, 2. Disagree, 3. Neither agree nor disagree, 4. Agree, 5. Strongly agree). They were also asked to rate Y_lra in percentage (0 to 100). From these variables, a multiple regression equation is formulated as

$\mathrm{Y}_{\mathrm{lra}}=\omega_{01}+\omega_{\mathrm{dcb} 1} \mathrm{X}_{\mathrm{dcb}}+\omega_{\mathrm{tcl} 1} \mathrm{X}_{\mathrm{tlc}}+\omega_{\mathrm{rdv} 1} \mathrm{X}_{\mathrm{rdv}}+\omega_{\mathrm{plc1}} \mathrm{X}_{\mathrm{plc}}+\omega_{\mathrm{sbc} 1} \mathrm{X}_{\mathrm{sbc}}$

Tab. 15 - Multiple regression output for a linear relationship between $\mathrm{Y}_{\mathrm{lra}}$ and $\mathrm{X}_{\mathrm{dcb}}, \mathrm{X}_{\mathrm{tlc}}, \mathrm{X}_{\mathrm{rdv}}$, $\mathrm{X}_{\mathrm{plc}}, \mathrm{X}_{\mathrm{sbc}}$. Source: Own

\begin{tabular}{|c|c|c|c|c|}
\hline \multicolumn{5}{|c|}{ Model Summary } \\
\hline Model & $\mathrm{R}$ & R Square & $\begin{array}{c}\text { Adjusted R } \\
\text { Square }\end{array}$ & $\begin{array}{c}\text { Std. Error of the } \\
\text { Estimate }\end{array}$ \\
\hline 1 & $.671^{\mathrm{a}}$ & .451 & .444 & 14.21163 \\
\hline
\end{tabular}

a. Predictors: (Constant), $\mathrm{X}_{\mathrm{dcb}}, \mathrm{X}_{\mathrm{tlc}}, \mathrm{X}_{\mathrm{rdv}}, \mathrm{X}_{\mathrm{plc}}, \mathrm{X}_{\mathrm{sbc}}$

\begin{tabular}{|c|c|c|c|c|c|c|}
\hline \multicolumn{7}{|c|}{ ANOVA $^{\circ}$} \\
\hline \multirow{2}{*}{ Model } & $\begin{array}{c}\text { Sum of } \\
\text { Squares }\end{array}$ & df & $\begin{array}{c}\text { Mean } \\
\text { Square }\end{array}$ & F & Sig. \\
\hline \multirow{3}{*}{1} & Regression & 65011.115 & 5 & 13002.223 & 64.377 & $.000^{a}$ \\
\cline { 2 - 8 } & Residual & 79172.352 & 392 & 201.970 & & \\
\cline { 2 - 8 } & Total & 144183.467 & 397 & & & \\
\hline
\end{tabular}

a. Predictors: (Constant), $\mathrm{X}_{\mathrm{dcb}}, \mathrm{X}_{\mathrm{tlc}}, \mathrm{X}_{\mathrm{rdv}}, \mathrm{X}_{\mathrm{plc}}, \mathrm{X}_{\mathrm{sbc}}$

b. Dependent Variable: $Y_{1 \mathrm{ra}}$ 


\begin{tabular}{|c|c|c|c|c|c|c|c|c|}
\hline \multicolumn{9}{|c|}{ Coefficients ${ }^{a}$} \\
\hline & \multirow{2}{*}{ Model } & \multicolumn{2}{|c|}{$\begin{array}{c}\text { Unstandardized } \\
\text { Coefficients }\end{array}$} & \multirow{2}{*}{$\begin{array}{c}\begin{array}{c}\text { Standardized } \\
\text { Coefficients }\end{array} \\
\text { Beta }\end{array}$} & \multirow[b]{2}{*}{$\mathrm{t}$} & \multirow[b]{2}{*}{ Sig. } & \multicolumn{2}{|c|}{$\begin{array}{c}95 \% \text { Confidence } \\
\text { Interval for B }\end{array}$} \\
\hline & & B & $\begin{array}{l}\text { Std. } \\
\text { Error }\end{array}$ & & & & $\begin{array}{l}\text { Lower } \\
\text { Bound }\end{array}$ & $\begin{array}{l}\text { Upper } \\
\text { Bound }\end{array}$ \\
\hline \multirow{6}{*}{1} & (Constant) & 49.911 & 3.277 & & 15.229 & .000 & 43.468 & 56.355 \\
\hline & $\mathrm{X}_{\mathrm{dcb}}$ & -9.470 & .673 & -.819 & -14.070 & .000 & -10.794 & -8.147 \\
\hline & $\mathrm{X}_{\mathrm{tlc}}$ & .463 & .697 & .037 & .664 & .507 & -.908 & 1.835 \\
\hline & $\mathrm{X}_{\mathrm{rdv}}$ & -.846 & .729 & -.045 & -1.161 & .246 & -2.279 & .587 \\
\hline & $\mathrm{X}_{\mathrm{plc}}$ & -1.396 & .648 & -.086 & -2.154 & .032 & -2.670 & -.122 \\
\hline & $\mathrm{X}_{\mathrm{sbc}}$ & 6.064 & .537 & .576 & 11.301 & .000 & 5.009 & 7.119 \\
\hline
\end{tabular}

a. Dependent Variable: $Y_{\text {lra }}$

From Table 15, we see that $\mathrm{X}_{\mathrm{dcb}}, \mathrm{X}_{\mathrm{tlc}}, \mathrm{X}_{\mathrm{rdv}}, \mathrm{X}_{\mathrm{plc}}$ and $\mathrm{X}_{\mathrm{sbc}}$ statistically significantly predict $\mathrm{Y}_{\text {lra }}$, $\mathrm{F}(5,392)=64.377, \mathrm{p}<0.05$. We can also see that $\mathrm{X}_{\mathrm{dcb}}, \mathrm{X}_{\mathrm{tc} c}, \mathrm{X}_{\mathrm{rdv}}, \mathrm{X}_{\mathrm{plc}}$ and $\mathrm{X}_{\mathrm{sbc}}$ explain $45.1 \%$ of the variability of $\mathrm{Y}_{\mathrm{lra}}$. We can therefore set a new regression equation as

$\mathrm{Y}_{\mathrm{lra}}=49.911-9.470 \mathrm{X}_{\mathrm{dcb}}+0.463 \mathrm{X}_{\mathrm{tlc}}-0.846 \mathrm{X}_{\mathrm{rdv}}-1.396 \mathrm{X}_{\mathrm{plc}}+6.064 \mathrm{X}_{\mathrm{sbc}}$

From the first hypothesis, $\left(\mathrm{H} 0: \omega_{\mathrm{dcb} 1}=0, \mathrm{H} 1: \omega_{\mathrm{dcb} 1} \neq 0\right)$, we reject $\mathrm{H} 0$; since $\omega_{\mathrm{dcb} 1}(-9.470)$ is statistically significantly different from $0(\mathrm{p}<0.05)$. There is a negative relationship between $\mathrm{X}_{\mathrm{dcb}}$ and $\mathrm{Y}_{1-}$ ${ }_{\mathrm{ra}}$. However, regarding the second hypothesis, $\left(\mathrm{H} 0: \omega_{\mathrm{tcc} 1}=0, \mathrm{H} 1: \omega_{\mathrm{tlc1}} \neq 0\right)$, we do not reject $\mathrm{H} 0$; since $\omega_{\text {tlc1 }}(0.463)$ is not statistically significantly different from $0(\mathrm{p}>0.05)$. Again, from the third hypothesis, $\left(\mathrm{H} 0: \omega_{\mathrm{rdv1}}=0, \mathrm{H} 1: \omega_{\mathrm{rdv} 1} \neq 0\right)$, we do not reject $\mathrm{H} 0$; since $\omega_{\mathrm{rdv} 1}(-0.846)$ is not statistically significantly different from $0(\mathrm{p}>0.05)$. But regarding the fourth hypothesis, $\left(\mathrm{H} 0: \omega_{\mathrm{plc}}=0, \mathrm{H} 1\right.$ : $\left.\omega_{\text {plc1 }} \neq 0\right)$, we reject $\mathrm{H} 0$; since $\omega_{\text {plc1 }}(-1.396)$ is statistically significantly different from $0(\mathrm{p}<0.05)$. A negative relationship between $\mathrm{X}_{\mathrm{plc}}$ and $\mathrm{Y}_{\text {lra }}$ is revealed. Also, from the fifth hypothesis, $(\mathrm{H} 0$ : $\left.\omega_{\text {sbc1 }}=0, H 1: \omega_{\text {sbc1 }} \neq 0\right)$, we reject $\mathrm{H} 0$; since $\omega_{\text {sbc1 }}(6.064)$ is statistically significantly different from $0(\mathrm{p}<0.05)$. We find a positive relationship between $\mathrm{X}_{\mathrm{sbc}}$ and $\mathrm{Y}_{\mathrm{lra}}$.

\section{Risk Reduction and Non-manufacturing Alliances}

In finding the relationship between non-manufacturing alliances and risk reduction, we tested the following hypotheses;

$$
\begin{aligned}
& \mathrm{H} 0: \lambda_{\text {jsl }}=0 \\
& \mathrm{H} 1: \lambda_{\mathrm{js}} \neq 0 \\
& \mathrm{H} 0: \lambda_{(\mathrm{dst})}=0 \\
& \mathrm{H} 1: \lambda_{(\mathrm{dst})} \neq 0 \\
& \mathrm{H} 0: \lambda_{\mathrm{lal}}=0 \\
& \mathrm{H} 1: \lambda_{\mathrm{lal}} \neq 0
\end{aligned}
$$


Where $\lambda_{0}, \lambda_{\mathrm{js}}, \lambda_{\mathrm{dst}}$, and $\lambda_{\mathrm{lal}}$ are coefficients for Constant, $\mathrm{X}_{\mathrm{js}}, \mathrm{X}_{\mathrm{dst}}$ and $\mathrm{X}_{\mathrm{lal}}$ respectively.

$\mathrm{X}_{\mathrm{jsl}}$ denotes "joint selling”

$\mathrm{X}_{\mathrm{dst}}$ denotes "joint distribution"

$\mathrm{X}_{\mathrm{lal}}$ denotes "learning alliances"

$\mathrm{Y}_{\text {lrr }}$ denotes "likelihood of risk reduction"

CEOs were asked to rate their level of agreement with $\mathrm{X}_{\mathrm{js}}, \mathrm{X}_{\mathrm{dst}}$ and $\mathrm{X}_{\mathrm{lal}}$ using a five-point scale (1. Strongly disagree, 2. Disagree, 3. Neither agree nor disagree, 4. Agree, 5. Strongly agree). They were also asked to rate $Y_{\text {lrr }}$ in percentage (0 to 100). From these variables, a multiple regression equation is formulated as

$\mathrm{Y}_{\mathrm{lrr}}=\lambda_{0}+\lambda_{\mathrm{jsl}} \mathrm{X}_{\mathrm{js}} 1+\lambda_{\mathrm{dst}} \mathrm{X}_{\mathrm{dst}}+\lambda_{\mathrm{lal}} \mathrm{X}_{\mathrm{lal}}$

Tab. 16 -Multiple regression output for a linear relationship between $\mathrm{Y}_{\mathrm{lrr}}$ and $\mathrm{X}_{\mathrm{js}}, \mathrm{X}_{\mathrm{dst}}, \mathrm{X}_{\mathrm{lal}}$. Source: Own

\begin{tabular}{|c|c|c|c|c|}
\hline \multicolumn{4}{|c|}{ Model Summary } \\
\hline Model & R & R Square & Adjusted R Square & Std. Error of the Estimate \\
\hline 1 & $.548^{\mathrm{a}}$ & .300 & .295 & 10.05971 \\
\hline
\end{tabular}

a. Predictors: (Constant), $\mathrm{X}_{\mathrm{js}}, \mathrm{X}_{\mathrm{dst}}, \mathrm{X}_{\mathrm{lal}}$

\begin{tabular}{|c|c|c|c|c|c|c|}
\hline \multicolumn{2}{|c|}{ ANOVA $^{\mathrm{b}}$} \\
\hline \multicolumn{2}{|c|}{ Model } & Sum of Squares & df & Mean Square & F & Sig. \\
\hline \multirow{3}{*}{1} & Regression & 17081.231 & 3 & 5693.744 & 56.263 & $.000^{\mathrm{a}}$ \\
\cline { 2 - 7 } & Residual & 39871.957 & 394 & 101.198 & & \\
\cline { 2 - 8 } & Total & 56953.188 & 397 & & & \\
\hline
\end{tabular}

a. Predictors: (Constant), $\mathrm{X}_{\mathrm{js}}, \mathrm{X}_{\mathrm{dst}}, \mathrm{X}_{\mathrm{lal}}$

b. Dependent Variable: $Y_{\text {lrr }}$

\begin{tabular}{|c|c|c|c|c|c|c|c|c|}
\hline \multicolumn{9}{|c|}{ Coefficients $_{\mathrm{a}}$} \\
\hline & \multirow{2}{*}{ Model } & \multicolumn{2}{|c|}{$\begin{array}{c}\text { Unstandardized } \\
\text { Coefficients }\end{array}$} & \multirow{2}{*}{$\begin{array}{c}\begin{array}{c}\text { Standardized } \\
\text { Coefficients }\end{array} \\
\text { Beta }\end{array}$} & \multirow[b]{2}{*}{$\mathrm{t}$} & \multirow[b]{2}{*}{ Sig. } & \multicolumn{2}{|c|}{$\begin{array}{c}95 \% \text { Confidence } \\
\text { Interval for B }\end{array}$} \\
\hline & & $\mathrm{B}$ & $\begin{array}{l}\text { Std. } \\
\text { Error }\end{array}$ & & & & $\begin{array}{l}\text { Lower } \\
\text { Bound }\end{array}$ & $\begin{array}{l}\text { Upper } \\
\text { Bound }\end{array}$ \\
\hline \multirow{4}{*}{1} & (Constant) & 42.826 & 1.938 & & 22.095 & .000 & 39.015 & 46.636 \\
\hline & $\mathrm{X}_{\mathrm{js} \mathrm{l}}$ & -2.098 & .442 & -.215 & -4.749 & .000 & -2.967 & -1.230 \\
\hline & $\mathrm{X}_{\mathrm{dst}}$ & -4.131 & .416 & -.467 & -9.939 & .000 & -4.948 & -3.314 \\
\hline & $\mathrm{X}_{\mathrm{lal}}$ & 2.528 & .510 & .236 & 4.959 & .000 & 1.526 & 3.530 \\
\hline
\end{tabular}

a. Dependent Variable: $Y_{1 \mathrm{rr}}$ 
From Table 16, we see that $X_{-j \mathrm{ss}}, \mathrm{X}_{-\mathrm{dst}}$ and $\mathrm{X}_{-\mathrm{lal}}$ statistically significantly predict $\mathrm{Y}_{\mathrm{lrr}}, \mathrm{F}(3,394)=$ $56.263, \mathrm{p}<0.05$.We can also see that $\mathrm{X}_{-\mathrm{js} \text { s }}, \mathrm{X}_{-\mathrm{dst}}$ and $\mathrm{X}_{-\mathrm{lal}}$ explain $30 \%$ of the variability of $\mathrm{Y}_{\text {lrr }}$. We can therefore set a new regression equation as

$\mathrm{Y}_{\mathrm{lrr}}=42.826-2.098 \mathrm{X}_{\mathrm{jsl}}-4.131 \mathrm{X}_{\mathrm{dst}}+2.528 \mathrm{X}_{\mathrm{lal}}$

From the first hypothesis, (H0: $\left.\lambda_{\text {jsl }}=0, \mathrm{H} 1: \lambda_{\text {jsl }} \neq 0\right)$, we reject $\mathrm{H} 0$; since $\lambda_{\text {jsl }}(-2.098)$ is statistically significantly different from $0(\mathrm{p}<0.05)$. A negative relationship between $\mathrm{X}_{\text {isl }}$ and $\mathrm{Y}_{\text {lrr }}$ is found. Regarding the second hypothesis, $\left(\mathrm{H} 0: \lambda_{\text {(dst) }}=0, \mathrm{H} 1: \lambda_{\text {dst }} \neq 0\right)$, we reject $\mathrm{H} 0$; since $\lambda_{\text {dst }}(-4.131)$ is statistically significantly different from $0(\mathrm{p}<0.05)$. Again, there is a negative relationship between $\mathrm{X}_{\mathrm{dst}}$ and $\mathrm{Y}_{\mathrm{lrr}}$. Likewise, from the third hypothesis, $\left(\mathrm{H} 0: \lambda_{\mathrm{lal}}=0, \mathrm{H} 1: \lambda_{\mathrm{lal}} \neq 0\right)$, we reject $\mathrm{H} 0$; since $\lambda_{\text {lal }}(2.528)$ is statistically significantly different from $0(\mathrm{p}<0.05)$. We find a positive relationship between $\mathrm{X}_{\mathrm{lal}}$ and $\mathrm{Y}_{\mathrm{lrr}}$.

\section{DISCUSSION}

We have seen through PCA that both the "joint production" and "assembling contracts" are not part of (not explaining) the manufacturing alliances. However, "design collaboration", "technology licensing", "collaboration in research and development", "product licensing" and "subcontracting" are. In this context, we argue that MEs are sceptical about producing goods together with their partners. We also argue that production is perceived as a sensitive task that cannot easily be shared with business partners. This is because manufacturing MEs do not prefer to assemble products together or vest assembling powers in their partners. The reasons might be similar to those rejecting joint production due to the fact that "assembling together" is almost similar to "producing together". Also, both the "joint promotion" and the "service agreements" do not explain the non-manufacturing alliances. However, "joint selling", "joint distribution" and "learning alliances" do. Promoting together might require partners to forego a lot of individual partner's traits which to a great extent might be a source of competitive advantage. In this regard, MEs might seem not to 'sacrifice' their positions. We also argue that sharing the delivery of services such as logistical services might be risky especially when these service agreements include serving the partners' customers, a role that cannot easily be foregone by MEs. We conclude that their scepticism on this is mainly fostered by the fact that customers ought to be handled by the same partner that recruited them.

The MRA indicates that "design collaboration", "technology licensing", "collaboration in research and development", and "product licensing" are likely to reduce the costs should the partners adopt them. Furthermore, the results point out that accessing resources and reducing risks can be possible through subcontracting and learning alliances respectively. However, the results have indicated that partnering firms cannot reduce costs or access resources if they embark on subcontracting and design collaboration respectively. The same is applied to product licensing. Neither joint selling nor joint distribution can help firms to reduce risks. We argue that the abovementioned poor influence has been caused by factors such as quality problems, management control, risk issues, and confidentiality, to mention a few. 
It was found that there is no any relationship between "collaboration in research and development" and partnering firms' likelihood of accessing resources. This tells that manufacturing MEs do not expect the exchange or any access of resources if they team up in research and development. We argue that manufacturing MEs cannot afford the huge research and development costs; that is why, they adopt a joint research and development strategy to reduce costs. Had this collaboration become a long term agreement (ten to fifteen years), manufacturing firms would have accessed resources including skills and experience; but the most underlying challenges facing manufacturing MEs from least developed countries like Tanzania is long term survival. However, we can conclude that partnering firms can acquire the necessary skills through their learning alliances that will also lead to risk reduction as we have already noticed. But these risks cannot be reduced by both joint selling and joint distribution as we have already realized. We therefore argue that the nature of the product (fast-moving consumer goods or highly perishable products) to a great extent, affects the choice of a distribution channel. It also affects the selling strategy. The various challenges associated with joint selling and joint distribution that can distort the partner's competitive advantage, influence partnering firms to adopt a go-it-alone strategy on selling and distribution matters.

We argue that manufacturing MEs regard subcontracting as a way of accessing resources provided that the right partner who can do better in a particular area of operation is recruited. Also, our assumption is that, product licensing, technology licensing and design collaboration are vehicles used to attract expertise from the partnering firm(s); hence, the likelihood of cost reduction. However, the results have indicated that technology licensing among partnering firms has no relationship with resource accessibility. Our assumption is that, manufacturing MEs perceive technology licensing as a strategy that can only help them recruit 'intangible resources' that seem to be insignificant to their growth and survival chances. Although, in technology licensing, the licenser is in most cases expected to control the relationship, we argue that the presence of technology in the licensee's hands could guarantee the licensee a sense of 'full ownership'. We still argue that manufacturing MEs ought not to waive the significance of technology in business. One of the means to acquire technology is through technology licensing that would enable them reduce both costs and risks hence maximize their survival chances.

\section{CONCLUSION}

We have seen that there is a relationship between alliance typology and the likelihood of both cost and risk reductions as well as resource accessibility. Although not all types of alliances reduce the costs and risks, and enable the partnering firms acquire resources, we find that most of the alliances in the manufacturing industry can exert a great influence should the alliance partners carefully blend the right mix of costs, risks, and resources. For instance, there can be a direct relationship between costs and risks; and therefore, a carefully selected alliance type adopted by the partners can concurrently help them reduce both risks and costs. The same case applies to the relationship between risks, costs and resources especially when partners establish an alliance with the aim of acquiring resources that will eventually be used to reduce both costs and risks. 


\section{Acknowledgements}

I express my gratitude to Kusekwa S. Mabondo, Emmanuel Kilatu, and two anonymous reviewers from the Journal of Competitiveness for their useful comments and suggestions.

\section{References}

1. Al Khattab, S. A. (2012). Marketing Strategic Alliances: The Hotel Sector in Jordan. International Journal of Business and Management, 7(9), 222-232. http://dx.doi.org/10.5539/ijbm. v7n9p222

2. Barney, J. (1991). Firm Resources and Sustained Competitive Advantage. Journal of Management, 17 (1), 99-120. http://dx.doi.org/10.1177/014920639101700108

3. Chen, H. M., \& Tseng, C. H. (2005). The Performance of Marketing Alliances Between the Tourism Industry and Credit Card Issuing Banks in Taiwan. Tourism Management, 26, 15-24. http://dx.doi.org/10.1016/j.tourman.2003.08.018

4. Coopers and Lybrand. (1997). Strategic Alliances. Coopers and Lybrand Barometer.

5. Das, T. K., \& Teng, B.-S. (2000). A Resource-Based Theory of Strategic Alliances. Journal of Management, 26(1), 31-61. http://dx.doi.org/10.1177/014920630002600105

6. Dussauge, P., Garrette, B., \& Mitchell, W. (2004). Asymmetric Performance:The Market Share Impact of Scale and Link Alliances in the Global Auto Industry. Strategic Management Journal, 25, 701-711. http://dx.doi.org/10.1002/smj.416

7. Elmuti, D., Abebe, M., \& Nicolosi, M. (2005). An Overview of Strategic Alliances Between Universities and Corporations. The Journal of Workplace Learning , 17 (1/2), 115-129. http:// dx.doi.org/10.1108/13665620510574504

8. Elmuti, D., \& Kathawala, Y. (2001). An Overview of Strategic Alliances. Management Decision, 39(3), 205-217. http://dx.doi.org/10.1108/EUM0000000005452

9. Gulati, R. (1998). Alliances and Networks. Strategic Management Journal, 19(4), 293-317. http:// dx.doi.org/10.1002/(SICI)1097-0266(199804)19:4<293::AID-SMJ982>3.0.CO;2-M

10. Hagedoorn, J., Letterie, W., \& Palm, F. (2011). The Information Value of R\&D Alliances: The Preference for Local or Distant Ties. Strategic Organization, 9(4), 283-309. http://dx.doi. org $/ 10.1177 / 1476127011421535$

11. Helper, S., Krueger, T., \& Wial, H. (2012). Why Does Manufacturing Matter? Which Manufacturing Matters? A Policy Framework. Washington: Metropolitan Policy Program at Brookings.

12. IBM Corporation. (2008). Operational Excellence in Manufacturing: How to Win at the Margins with Business Intelligence. Ottawa: IBM Corporation.

13. Ireland, R. D., Hoskisson, R. E., \& Hitt, M. A. (2009). The Management of Strategy (8th ed.). Mason, OH: South Western Cengage Learning.

14. Išoraitè, M. (2009). Importance of Strategic Alliances in Company's Activity. Intellectual Economics , 1 (5), 39-46.

15. Kaiser, F. H. (1974). An Index of Factorial Simplicity. Psychometrika, 39, 32-36. 
16. Knoke, D. (2009). Playing Well Together:Creating Corporate Social Capital in Strategic Alliance Networks. American Behavioral Scientist, 52(12), 1690-1708.http://dx.doi.org/10.1177 /0002764209331533

17. Koza, M., \& Lewin, A. (2000). Managing Partnerships and Strategic Alliances:Raising the Odds of Success. European Management Journal, 18(2), 146-151.http://dx.doi.org/10.1016/ S0263-2373(99)00086-9

18. Estélyiová, K. (2012). Governance Structure of Strategic Alliances-Evidences From the South Moravian Region. Journal of Competitiveness, 4(2), 172-184. http://dx.doi.org/10.7441/ joc.2012.02.12

19. Livesey, F. (2006). Defining High Value Manufacturing. Mill Lane, Cambridge: University of Cambridge Institute for Manufacturing.

20. Martins, R., Serra, F. R., Leite, A. S., Ferreira, M. P., \& Li, D. (2010). Transactions Cost Theory Influence in Strategy Research: A Review Through a Bibliometric Study in Leading Journals. Leiria: Glob Advantage-Center of Research in International Business \& Strategy.

21. Mills, J., \& Platts, K. (2003). Applying Resource-based Theory:Methods, Outcomes and Utility for Managers. International Journal of Operations \& Production Management, 23 (2), 148 166. http://dx.doi.org/10.1108/01443570310458429

22. Mowla, M. M. (2012). An Overview of Strategic Alliance: Competitive Advantages in Alliance Constellations. Journal of Business Management and Corporate Affairs , 1 (1), 1-10.

23. NBS. (2013). Quarterly Production of Industrial Commodities: 2004-2012. Dar es Salaam: National Bureau of Statistics, United Republic of Tanzania.

24. Paik, Y. (2005). Risk Management of Strategic Alliances and Acquisitions between Western MNCs and Companies in Central Europe. Thunderbird International Business Review, 47(4), 489511.http://dx.doi.org/10.1002/tie.20064

25. Sheppard, J. P. (1995). A Resource Dependence Approach to Organizational Failure. Social Science Research, 24, 28-62.

26. Singh, R. (2006). Introduction to Basic Manufacturing Processes and Workshop Technology. New Delhi: New Age International (P) Ltd.

27. Todeva, E., \& Knoke, D. (2005). Strategic Alliances and Models of Collaboration. Management Decision, 43(1), 123-148. http://dx.doi.org/10.1108/00251740510572533

28. URT. (2003). Small and Medium Enterprise Development Policy. Dar es Salaam: Ministry of Industry and Trade - The Government of the United Republic of Tanzania.

29. Van Gils, A., \& Zwart, P. S. (2009). Alliance Formation Motives in SMEs:An Explorative Conjoint Analysis Study. International Small Business Journal, 27(1), 5-37. http://dx.doi. $\operatorname{org} / 10.1177 / 0266242608098345$

30. Wernefelt, B. (1984). A Resource-based View of the Firm. Strategic Management Journal, 5, 171180. http://dx.doi.org/10.1002/smj.4250050207

31. Zamir, Z., Sahar, A., \& Zafar, F. (2014). Strategic Alliances; A Comparative Analysis of Successful Alliances in Large and Medium Scale Enterprises around the World. Educational Research International , 3 (1), 25-39. 
32. Zineldin, M., \& Dodourova, M. (2005). Motivation, Achievements and Failure of Strategic Alliances: The Case of Swedish Auto-Manufacturers in Russia. European Business Review, 17 (5), 460-470. http://dx.doi.org/10.1108/09555340510620357

\section{Contact Information}

Kafigi Jeje

Institute of Accountancy Arusha, Department of Business Management

Po Box 2798 Arusha, Tanzania

Email:kjeje@iaa.ac.tr. 